\title{
Channel equalization using watermark as a training sequence
}

\author{
M. K. Samee and J. Götze \\ Information Processing Lab, Dortmund University of Technology, Otto-Hahn-Str. 4, 44227 Dortmund, Germany
}

\begin{abstract}
In this paper blind equalization algorithms based on digital watermarking are presented. Training sequence is sent along with the data in the form of watermark. Robust CDMA based watermarking algorithm is used to watermark the data. Watermark, which is spread over the data, does not consume extra bandwidth. So with the help of extracted watermark channel can be equalized just by using simple mean square algorithm. Two algorithms for equalization are proposed in this paper. In algorithm I the receiver does not require to know training sequence in advance. Using algorithm II, the sender does not require to send training sequence either, and data can still be equalized using simple LMS algorithm at low computational complexity.
\end{abstract}

\section{Introduction}

Intersymbol Interference (ISI) is a very common type of distortion which a signal undergoes during transmission (Proakis, 2000). Trained equalization is a technique widely used to cope with ISI. A training sequence is sent over the channel and with the help of received and already known training sequence channel is equalized. Traditional trained equalization scheme works with simple algorithms like Least Mean Squares (LMS) (Haykin, 1996). Most crucial drawback of traditional trained equalization is that it consumes extra bandwidth. To save the bandwidth, some blind equalization techniques, which do not require training sequences, are used to equalize channels. These blind equalization schemes are usually based on computationally complex algorithms like Constant Modulus Algorithm (CMA) (Litwin, 1999). So the cost of saving bandwidth is increased computational complexity.

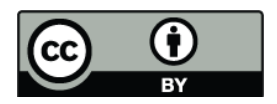

Correspondence to: $\mathrm{M}$. K. Samee (kashif.samee@tu-dortmund.de)
Digital Watermarking is a well known technique used for authentication, copyrights protection and applications like avoiding illicit copying etc. Watermark is simply a sequence of some bits which is hidden in host data (e.g. videos, images and someother multimedia data). If some meaningful watermark is embedded in host data watermarking can be used for some other purposes as well. Tracing watermarks are used for blind quality assessment for multimedia communication in (Campisi, 2003). A multipurpose public-key cryptosystem based on image watermarking is developed in (Ding, 2008). End-to-end QoS provision and control in wireless communication by means of digital watermarking is presented in (Benedetto, 2006). Digital watermarking is used for dynamic and adaptive equalization in (Maity, 2007; Neto, 2006) respectively. However in (Maity, 2007; Neto, 2006) receiver should know the watermark (training sequence) in advance, which is not required in proposed scheme.

In comparison to blind equalization, traditional trained equalization is known to be computationally efficient. But the most crucial drawback of trained equalization is that it consumes extra bandwidth. Another disadvantage is that the receiver must have prior information of the original training sequence. There are some blind equalization algorithms, where training sequences are not used and data can still be equalized (Litwin, 1999; Zarzoso, 2005). To design a blind equalization algorithm convergence of the algorithm and computational complexity are two important issues. Constant Modulus Algorithm (CMA) (Litwin, 1999) is one of well known algorithm used for blind equalization. Disadvantage for CMA is its slow convergence. Therefore, researchers tried to lessen the computational complexity of CMA and tried to make convergence faster (Sun, 2007; Dabeer, 2003).

In this work, a completely different approach to blind equalization is taken by using digital watermarking. The proposed method does not require training sequences to be known in advance and works with standard trained equalization algorithms (e.g. LMS) at low computational complexity.

Published by Copernicus Publications on behalf of the URSI Landesausschuss in der Bundesrepublik Deutschland e.V. 
Furthermore, proposed scheme can simultaneously be used for blind equalization and usual watermarking applications (Podilchuk, 2001).

Proposed schemes can be looked from two different viewpoints. First, how watermarking can be used for blind equalization (the receiver is not required to know the training sequence). Second, how digital watermarking can be used to correct errors in transmitted images. Additionally the proposed algorithms can simultaneously be used for blind equalization and usual watermarking applications. In this paper, two watermarking based equalization algorithms are presented, for two different scenarios:

1. when channel specific training sequence or specific watermark is required,

2. when watermark/training sequence can be any sequence (can be a part of data).

In algorithm I, training sequence is embedded in the data in the form of watermark. This training sequence is also sent over the channel. On receiving side, the watermark is extracted and with the help of received training sequence and extracted watermark channel can be equalized. In this algorithm receiver does not required to know the training sequence in advance. Consider the following scenario; An image (data) distributor distributes watermarked images (data) containing different watermarks to several clients. Distributor also sends the watermark along with the watermarked image (data) through the channel. On the receiving sides, receivers only have a watermark extractor (all the receivers use the same extracting software). Therefore, the receivers can extract the watermark and use it as a reference training sequence. The watermark which distributor has sent through the channel can be treated as received training sequence. Now, the channel can be estimated and hence received, erroneous, watermarked images can be corrected.

In algorithm II, a chunk of data, which is a part of the data actually to be transmitted through the channel, is used as a watermark. This chunk is embedded in the data as a watermark. With the help of received chunk and extracted chunk (extracted watermark) the channel can be equalized blindly. Watermarking algorithm used for these blind equalization schemes, must have the following two qualities:

- Robust (to withstand distortions due to transmission).

- Carry enough payload (which can be used as training sequence).

CDMA based spread spectrum watermarking scheme (Samee, 2007) fulfills the above requirements. The main advantage of spread spectrum watermarking is that each watermark bit is embedded in a number of pixels. Because of that it is proved to be robust in transmission. By using multiple orthogonal spreading codes, CDMA based watermarking scheme can carry more payload (large watermark). Normalized Least Mean Square (NLMS) algorithm (Haykin, 1996) is used in this scheme for equalization. Other equalization algorithms e.g. Least Mean Square (LMS) and Recursive Least Square (RLS) can also be used.

This paper is organized as follows: Sect. 2 presents an overview of previously described CDMA based watermarking algorithm. In Sect. 3 blind equalization scheme using digital watermarking is described. Section 4 presents the experimental results and Sect. 5 concludes this paper.

\section{Overview of the watermarking scheme}

An oblivious watermarking scheme is presented in (Samee, 2007), which is further improved by using by-part interleaving in (Samee, 2008). In this watermarking scheme every bit of the watermark is hidden in mutually similar frequency coefficients in the DCT domain. These specially selected frequency coefficients are preferably in middle frequency range. Every vector of the modified frequency coefficients $\boldsymbol{i}_{j}^{\prime}$ is formed according to:

$\boldsymbol{i}_{j}^{\prime}=\boldsymbol{i}_{j}+\alpha\left[b_{1} \boldsymbol{s}_{\mathbf{1}}+b_{2} \boldsymbol{s}_{\mathbf{2}}+\ldots+b_{k} \boldsymbol{s}_{\boldsymbol{k}}\right]$

where $\boldsymbol{i}_{\boldsymbol{j}}$ is a vector of similar frequency coefficients, $b_{i}$ is a watermark bit, $\alpha$ is the gain factor and $s_{i}$ are the spreading codes. Watermark bits can be extracted by using:

$b_{i}=\operatorname{sign}<\boldsymbol{i}_{j}^{\prime}, \boldsymbol{s}_{\boldsymbol{i}}>\quad$ if $\left|\boldsymbol{i}_{\boldsymbol{j}} \cdot \boldsymbol{s}_{\boldsymbol{i}}^{\boldsymbol{T}}\right|<\left|\alpha \boldsymbol{s}_{\boldsymbol{i}} \cdot \boldsymbol{s}_{\boldsymbol{i}}^{\boldsymbol{T}}\right|$

For complete description of the watermarking scheme see (Samee, 2007). This scheme is proved to be robust against many intentional and unintentional attacks. Transmission of watermarked image through a channel can be seen as an unintentional attack. The watermark easily survives the transmission attack by using this watermarking scheme. The use of multiple spreading codes makes this scheme able to carry large watermark.

\section{Watermarking-based blind equalization}

Figure 1 shows how trained equalization works. In order to equalize the data receiver must know the reference training sequence in advance. Error $e(n)$ is calculated with the help of received training sequence and reference training sequence. Now by minimizing $e(n)$, the inverse of the channel is estimated and weights (taps) $\boldsymbol{w}(n)$ are updated. The received data can be equalized by using $\boldsymbol{w}(n)$.

Figure 2 shows algorithm I for blind equalization, where a training sequence is hidden (or superimposed) in the data by using digital watermarking. After transmission, if this hidden training sequence is extracted from the received data without much errors, the extracted training sequence can be used as reference training sequence (which is supposed to be provided by the sender). 


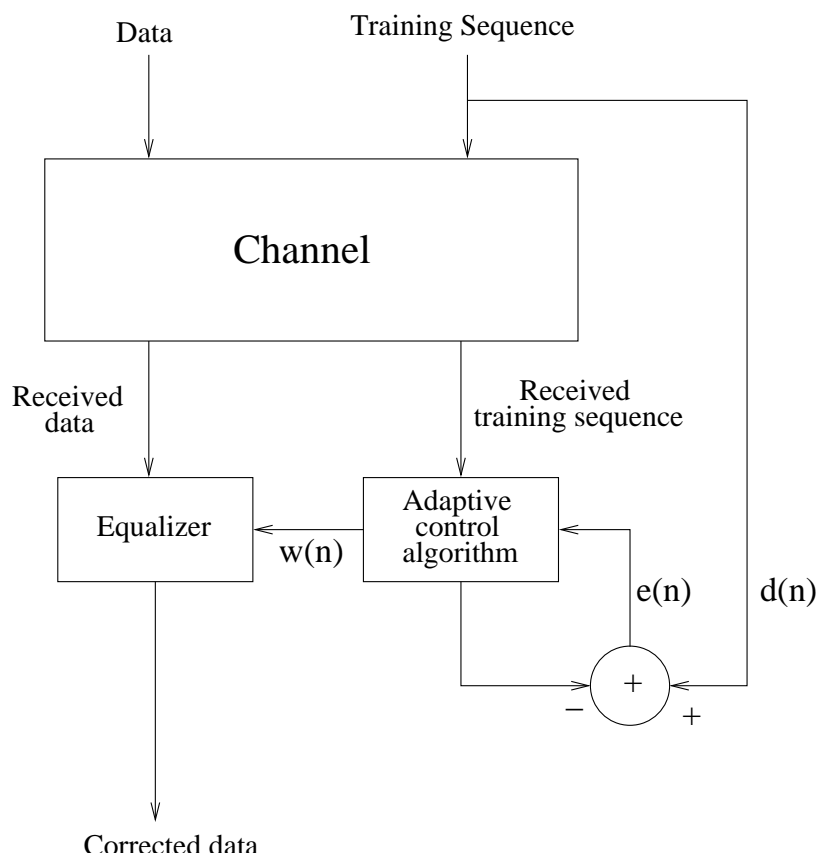

Fig. 1. Traditional trained equalization technique.

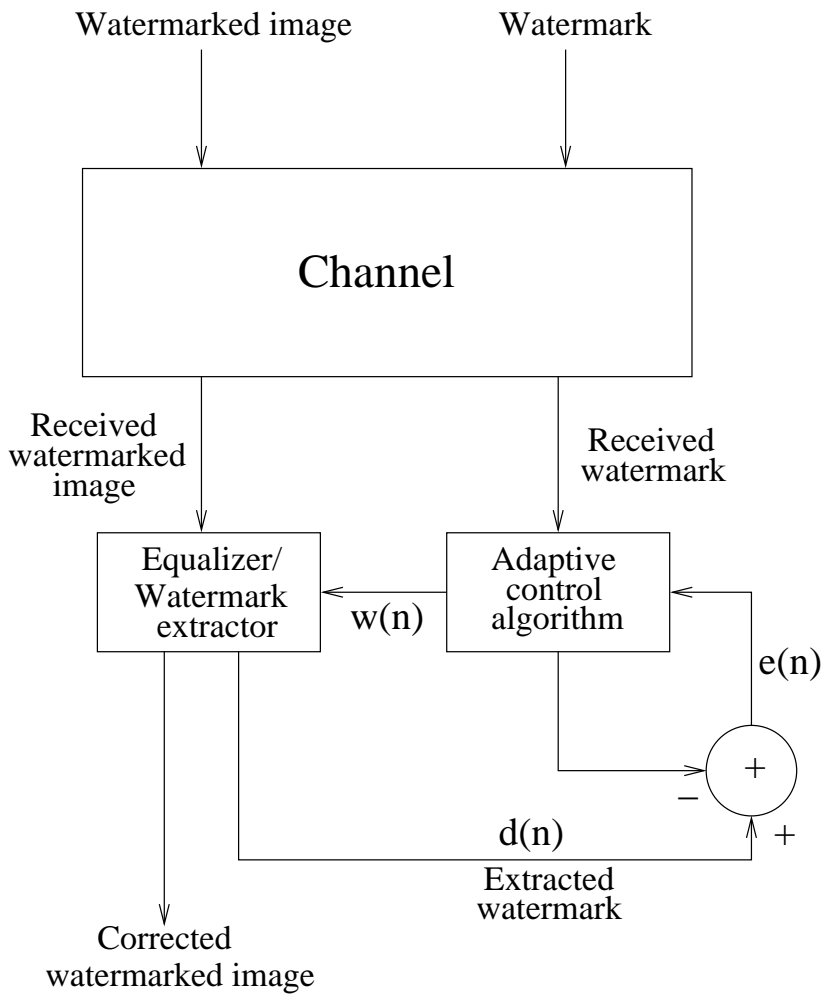

Fig. 2. Algorithm I for blind equalization.

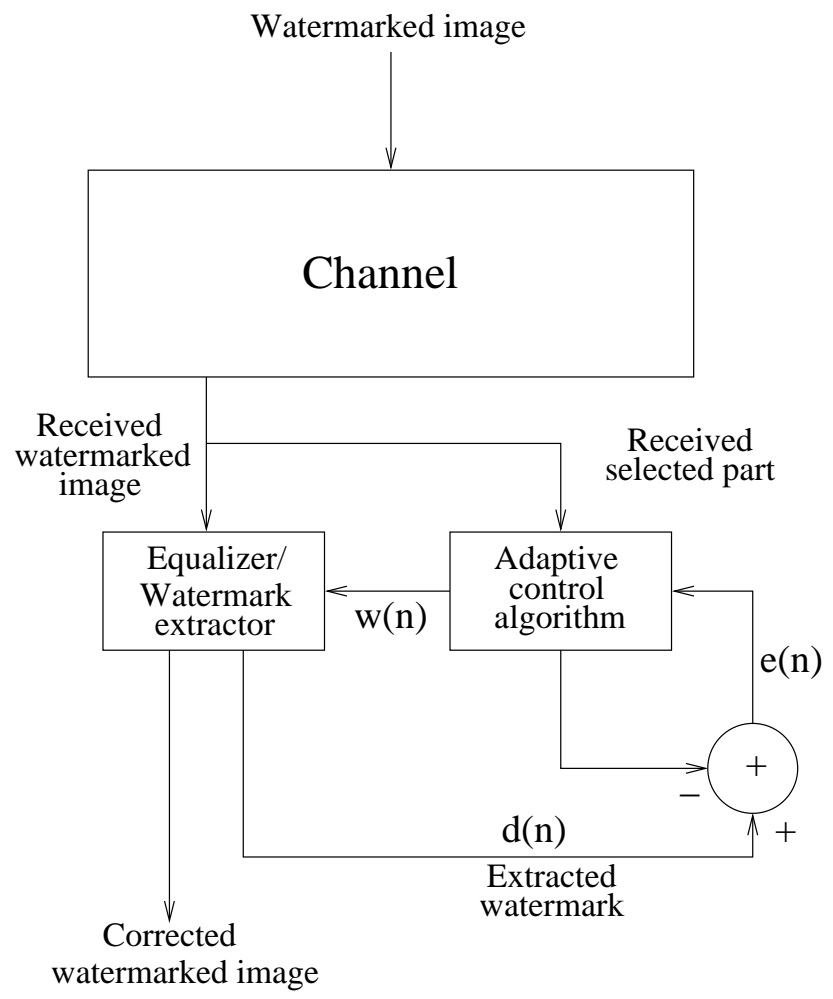

Fig. 3. Algorithm II for blind equalization.

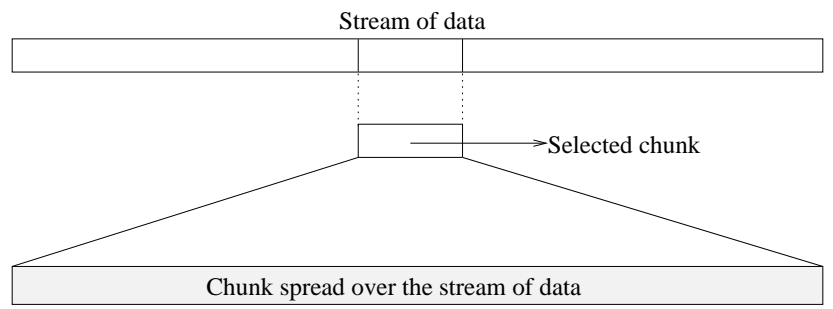

Fig. 4. Selecting a chunk of data and hidding it in the stream of data.

Figure 3 shows algorithm II for blind equalization, where a selected chunk of data is hidden (or superimposed) in the entire stream of data (Fig. 4 ). After transmission, this hidden chunk of data is extracted from the received data. This extracted chunk can be used as a reference training sequence and the received chunk can be used as a received training sequence. Thus $e(n)$ can be calculated and NLMS algorithm can be used to update $\boldsymbol{w}(n)$. Hence received data can be equalized blindly.

For equalization Normalized LMS algorithm is used. This algorithm works as follows Haykin (1996): 


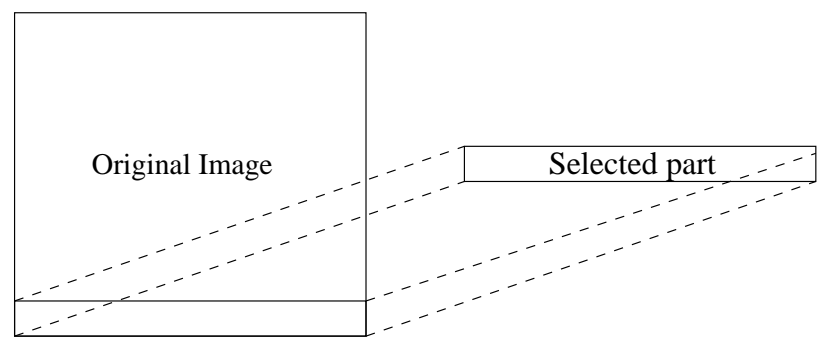

Table 1. BER in extracted watermarks using algorithm I.

\begin{tabular}{ccccc}
\hline channels & \multicolumn{2}{c}{ Received images } & \multicolumn{2}{c}{ Equalized images } \\
$\%$ & Milk drop & Lena & Milk drop \\
$\%$ & $\%$ & $\%$ \\
\hline 1 & 8.2 & 8.59 & 0 & 0 \\
2 & 16.8 & 16.8 & 0 & 0 \\
3 & 0.39 & 10.16 & 0 & 0 \\
4 & 2.54 & 10.55 & 0 & 0 \\
\hline
\end{tabular}

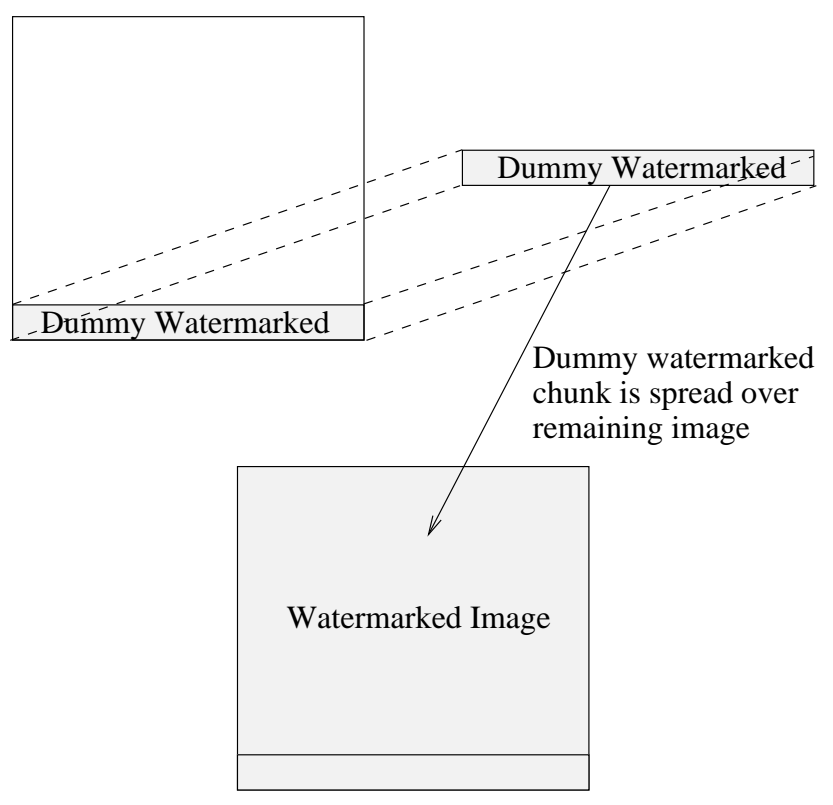

Fig. 5. Forming a watermarked image, considering implementation problem.

$$
\text { Parameter: } \begin{aligned}
M & =\text { number of taps } \\
a & =\text { positive constant } \\
\tilde{\mu} & =\text { adaption constant (step-size parameter) } \\
0 & <\tilde{\mu}<2
\end{aligned}
$$

Initialization: if prior knowledge on the tap-weight vector $\hat{\boldsymbol{w}}(n)$ is available, use it to select an appropriate value for $\hat{\boldsymbol{w}}(0)$. Otherwise, set $\hat{\boldsymbol{w}}(0)=\mathbf{0}$.

Data:

(a) Given: $\boldsymbol{u}(n)$ : M-by-1 tap-input vector at time $\mathrm{n}$ $d(n):$ desired response at time $\mathrm{n}$

(b) To be computed: $\hat{\boldsymbol{w}}(n+1)=$ estimate of tap-weight vector at time $n+1$
Computation $: n=0,1,2, \ldots$

$$
\begin{aligned}
e(n) & =d(n)-\hat{\boldsymbol{w}}^{H}(n) \boldsymbol{u}(n) \\
\hat{\boldsymbol{w}}(n+1) & =\hat{\boldsymbol{w}}(n)+\frac{\tilde{\mu}}{a+\|\boldsymbol{u}(n)\|^{2}} \boldsymbol{u}(n) e^{*}(n)
\end{aligned}
$$

Normalized LMS is a modified version of LMS algorithm. LMS algorithm also works fine in proposed blind equalization method. However simulations have shown that Normalized LMS algorithm works slightly better than LMS algorithm. LMS algorithm experiences a gradient noise amplification problem when $\boldsymbol{u}(n)$ is large, because the correction applied to the tap-weight vector $\hat{\boldsymbol{w}}(n)$ at iteration $n+1$ is directly proportional to the tap-input vector $\boldsymbol{u}(n)$. In normalized LMS algorithm correction applied to tap-weight vector $\hat{\boldsymbol{w}}(n)$ at iteration $n+1$ is normalized with respect to squared Euclidean norm of tap-input vector $\boldsymbol{u}(n)$ at iteration $n$. That is why it is called "normalized" LMS.

\subsection{Implementation problem}

A very crucial problem arose while implementing proposed scheme using digital watermarking. A part of an image is selected, and later it is spread over the whole image forming watermarked image. Obviously, watermarked image is slightly different from the original image. When this watermarked image is transmitted, channel can not be equalized. Because, the extracted watermark is a part of the original image and received part is from watermarked image. Hence both of them are different and the channel can not be equalized using extracted watermark and received part of the watermarked image.

One of the simplest solutions to this problem is to hide the selected part in the remaining image (other than selected part). However, this simple solution causes problems for usual watermarking applications, like copyrights protection etc. From general watermarking applications point of view, it is not good to leave a reference to the original image. Watermark should be spread over the whole image. If reference to the original image is present in the watermarked image, some denoising algorithm can detect watermark as noise. So watermark can be removed easily. Therefore, this scheme can not be used for usual watermarking applications. 
Table 2. Lena received and equalized using algorithm I.

\begin{tabular}{ccccc}
\hline \multirow{2}{*}{ channels } & \multicolumn{2}{c}{ PSNR } & \multicolumn{2}{c}{ Erroneous bits } \\
& $\begin{array}{c}\text { Received } \\
\mathrm{dB}\end{array}$ & $\begin{array}{c}\text { Equalized } \\
\mathrm{dB}\end{array}$ & $\begin{array}{c}\text { Received } \\
\%\end{array}$ & $\begin{array}{c}\text { Equalized } \\
\%\end{array}$ \\
\hline 1 & 29.0929 & 77.5595 & 8.94 & 0.01 \\
2 & 28.9833 & 69.6869 & 12.2 & 0.09 \\
3 & 22.0178 & Infinite & 33.58 & 0.00 \\
4 & 13.8834 & 42.9292 & 9.29 & 0.00 \\
\hline
\end{tabular}

Table 3. Milk drop received and equalized using algorithm I.

\begin{tabular}{ccccc}
\hline \multirow{2}{*}{ channels } & \multicolumn{2}{c}{ PSNR } & \multicolumn{2}{c}{ Erroneous bits } \\
& Received & Equalized & Received & Equalized \\
& $\mathrm{dB}$ & $\mathrm{dB}$ & $\%$ & $\%$ \\
\hline 1 & 29.2870 & 74.1472 & 8.51 & 0.03 \\
2 & 29.1856 & 92.3162 & 11.65 & 0.00 \\
3 & 23.0038 & Infinite & 33.58 & 0.00 \\
4 & 13.8618 & 21.8373 & 9.29 & 1.27 \\
\hline
\end{tabular}

This problem is solved by watermarking selected portion with some dummy bits first and then hide this dummy watermarked selected portion into remaining image (Fig. 5). Now this watermarked image has same level of noise over whole image and has no reference to the original image. Therefore, this watermarked image can simultaneously be used for blind equalization as well as for usual watermarking applications.

\subsection{Possible security issue}

As watermark is a part of the watermarked image, it can be considered as a possible security issue. By using proposed scheme, watermark can be any part of watermarked image, which is very difficult to locate for an evedropper. Furthermore, because of proposed implementation scheme noise level (watermark intensity) is same over the whole image. So it is difficult to distinguish between selected part, watermark, and remaining watermarked image.

\section{Experimental results}

In all the experiments $512 \times 512$ ( 8 bits/pixel, gray scale) images (Lena Fig. 6a and Milk drop Fig. 6b) are used. 512 bits long watermark is hidden in $3 \mathrm{rd}, 4 \mathrm{th}, 5$ th and 6 th rows of $8 \times 8$ blocks of DCT coefficients (Samee, 2007). Two spreading codes, each of length 512, are used to spread the watermark bits. All the images are watermarked at $40 \mathrm{~dB}$ (here $\alpha=0.01)$. The quality of an image is measured in terms of Peak Signal to Noise Ratio (PSNR).
Table 4. BER in extracted watermarks using algorithm II.

\begin{tabular}{ccccc}
\hline \multirow{2}{*}{ channels } & \multicolumn{2}{c}{ Received images } & \multicolumn{2}{c}{ Equalized images } \\
& $\%$ & Milk drop & Lena & Milk drop \\
$\%$ & $\%$ & $\%$ \\
\hline 1 & 4.76 & 8.93 & 0 & 0 \\
2 & 6.75 & 10.52 & 0 & 0 \\
3 & 0.2 & 4.37 & 0 & 0 \\
4 & 24.40 & 17.26 & 0 & 0 \\
\hline
\end{tabular}

Table 5. Lena received and equalized using algorithm II.

\begin{tabular}{ccccc}
\hline \multirow{2}{*}{ channels } & \multicolumn{2}{c}{ PSNR } & \multicolumn{2}{c}{ Erroneous bits } \\
& Received & $\begin{array}{c}\text { Equalized } \\
\mathrm{dB}\end{array}$ & $\mathrm{dB}$ & Received \\
$\%$ & $\begin{array}{c}\text { Equalized } \\
\%\end{array}$ \\
\hline 1 & 26.8461 & 87.1311 & 9.67 & 0.00 \\
2 & 26.8677 & 86.5184 & 13.04 & 0.00 \\
3 & 22.5406 & 102.3162 & 33.60 & 0.00 \\
4 & 12.3285 & 88.8920 & 8.06 & 0.00 \\
\hline
\end{tabular}

PSNR $=10 \log \frac{255^{2}}{\mathrm{MSE}} \mathrm{dB}$

Using MATLAB, these images are transmitted over four different channels:

channel 1: $\quad\{0.986,0.845,0.237,0.123+0.310 \mathrm{i}\}$

channel 2: $\quad\{0.986,0.845,0.537,0.323,0.123\}$

channel 3: Frequency-flat (single path) Rayleigh fading channel, sample time $1 \times 10^{-5}$ and maximum Doppler shift $0.09 \mathrm{~Hz}$.

channel 4: Frequency-flat (single path) Rician fading channel, sample time $1 \times 10^{-5}$, maximum Doppler shift $0.99 \mathrm{~Hz}$ and Rician factor equal to 1.

\subsection{Algorithm I}

First of all, training sequence is embedded in an image as watermark. 512 bits long training sequence (watermark) is sent over the channel followed by the watermarked image. On the receiving side, watermark is extracted from the watermarked image. The extracted watermark has a few erroneous bits (table 1) but the BER is good enough to use it as a reference training sequence. Normalized LMS with 8 weights, $\tilde{\mu}=0.01$ and $\hat{\boldsymbol{w}}(0)=\mathbf{0}$ are used. The calculated weights $\hat{\boldsymbol{w}}(\boldsymbol{n})$ are used to equalize the received image. Received and corresponding equalized Lena images are shown in Fig. 7. Errors and PSNR values of received and equalized 
Table 6. Milk drop received and equalized using algorithm II.

\begin{tabular}{ccccc}
\hline channels & \multicolumn{2}{c}{ PSNR } & \multicolumn{2}{c}{ Erroneous bits } \\
& $\begin{array}{c}\text { Received } \\
\mathrm{dB}\end{array}$ & $\begin{array}{c}\text { Equalized } \\
\mathrm{dB}\end{array}$ & $\begin{array}{c}\text { Received } \\
\%\end{array}$ & $\begin{array}{c}\text { Equalized } \\
\%\end{array}$ \\
\hline 1 & 27.2704 & 83.6830 & 4.56 & 0.00 \\
2 & 27.3037 & 74.7575 & 4.76 & 0.03 \\
3 & 22.6232 & 102.3162 & 10.32 & 0.00 \\
4 & 13.2914 & 102.3162 & 14.88 & 0.00 \\
\hline
\end{tabular}

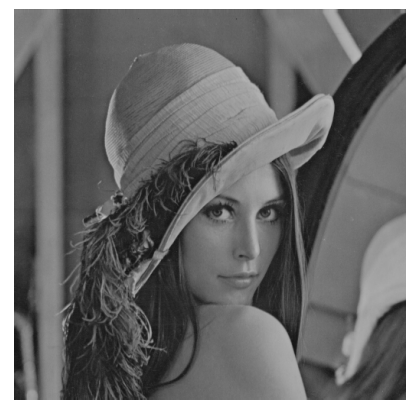

(a) Lena image.

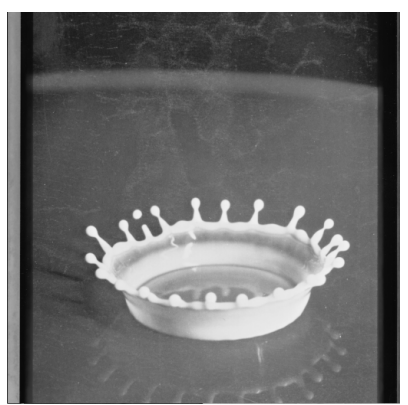

(b) Milk drop.

Fig. 6. Original Lena and Milk drop images.

images for both Lena and Milkdrop are shown in Tables 2 and 3, respectively. Here, second and third columns show PSNR value of received and equalized images with respect to watermarked images. Fourth and fifth columns show percentage of erroneous bits in received and equalized images. Column 4 and 5 of Table 1 show the BER in the watermarks extracted from equalized images. These watermarks are error free and easily be used for usual watermarking applications.

\subsection{Algorithm II}

First eight rows of an image are watermarked with eight dummy bits. First 504 bits are selected from the dummy watermarked part and hidden in remaining rows of the image. These watermarked images (watermarked at $40 \mathrm{~dB}$ ) are transmitted over the same four channels, mentioned above.

On the receiving side, watermark is extracted from the watermarked image. The watermark extracted from the received image, which forms the training sequence, has a few erroneous bits (column 2 and 3 of Table 4). The received selected part is treated as the received training sequence. $\hat{\boldsymbol{w}}(n)$ are calculated using Normalized LMS with $M=8$ weights, step size $\tilde{\mu}=0.01$ and $\hat{\boldsymbol{w}}(0)=\mathbf{0}$. The calculated weights $\hat{\boldsymbol{w}}(n)$ are used to equalize the received image. Errors and PSNR values of received and corresponding equalized images are shown in Tables 5 and 6. Here, second and third columns show PSNR value of received and equalized images with respect to watermarked images. Fourth and fifth columns show percentage of erroneous bits in received and equalized images. Column 4 and 5 of Table 4 show the BER in the watermarks extracted from equalized images.

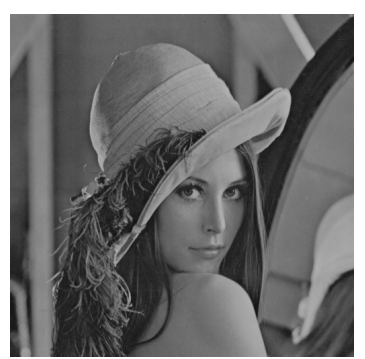

(a) Lena image.

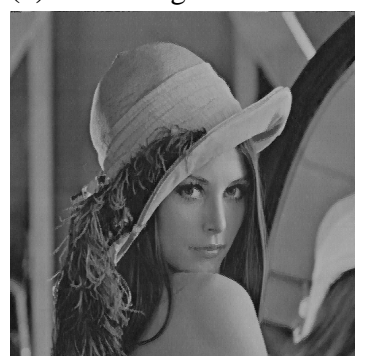

(c) Received through channel 1 .

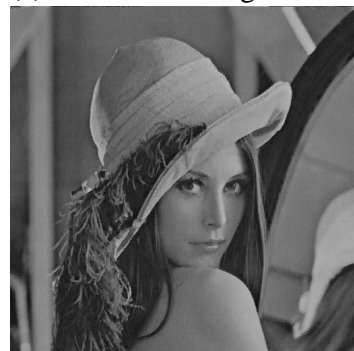

(e) Received through channel 2.

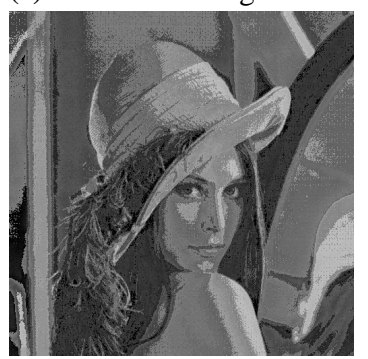

(g) Received through channel 3.

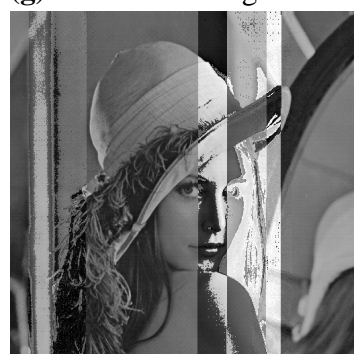

(i) Received through channel 4.

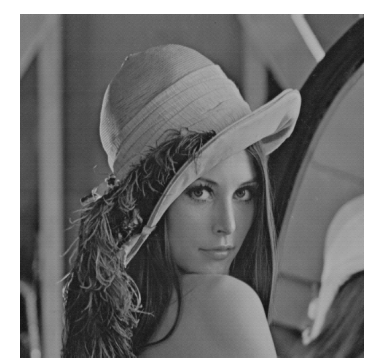

(b) Watermarked Lena.

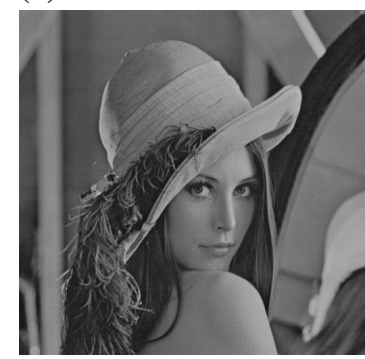

(d) Equalized image for (c)

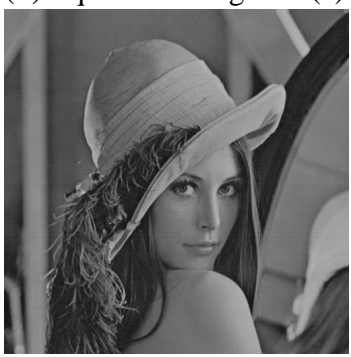

(f) Equalized image for (e).

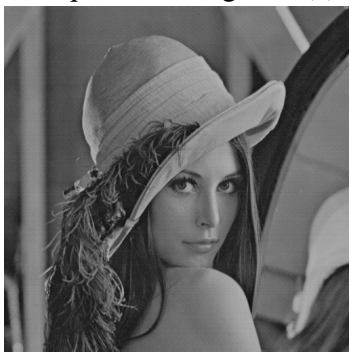

(h) Equalized image for (g).

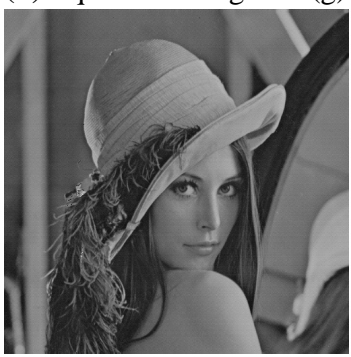

(j) Equalized image for (i).
Fig. 7. Lena received and equalized. 


\section{Conclusions}

How watermarking can be used to equalize transmission channels only using the received data is discussed in this paper. Simulations have shown that proposed schemes can correct almost all the errors from received watermarked images. An important advantage of this scheme is that it works at the complexity of traditional trained equalization methods unlike other very complex blind equalization methods. Proposed schemes can simultaneously be used for blind equalization as well as for usual watermarking applications. Further research will be done to develop a similar scheme for radio frequency communication signals. Furthermore, the suitability of different watermarking schemes for the proposed blind equalization algorithm can be studied.

\section{References}

John G. Proakis: Digital Communications, fourth edition, McGraw Hill, 2000.

Simon Haykin: Adaptive Filter Theory, third edition, Prentice Hall, 1996.

Litwin Jr., L. R.: Blind Channel Equalization, IEEE Potentials, 18(4), 9-12, 1999.

Campisi, P., Carli, M., Giunta, G., and Neri, A.: Blind Quality Assessment System for Multimedia Communications Using Tracing Watermarking, IEEE Transactions on Signal Processing [see also IEEE Transactions on Acoustics, Speech, and Signal Processing], 51(4), 996-1002, 2003.

Ding, Y. W., Lin, Z., and Wang, L.: A Multipurpose PublicKey Cryptosystem Based Image Watermarking, 4th International Conference on Wireless Communications, Networking and Mobile Computing, WiCOM '08., 2008.
Benedetto, F., Giunta, G., and Neri, A.: End-to-End QoS Provision and Control in Wireless Communication Systems by Means of Digital Watermarking Signal Processing, 3rd International Symposium on Wireless Communication Systems, ISWCS '06. 655659, 2006.

Maity, S. P., Kundu, M. K., and Maity, S.: An Efficient Digital Watermarking Scheme for Dynamic Estimation of Wireless Channel Condition, International Conference on Computing: Theory and Applications. ICCTA '07, 671-675, 2007.

Neto, M. U., Gomes, L. de C. T., Romano, J. M. T., and Bonnet, M.: Adaptive Equalization based on Watermarking, 2006 International Telecommunications Symposium, 743-748, 2006.

Zarzoso, V., and Comon, P.: Blind and Semi-Blind Equalization Based on the Constant Power Criterion, IEEE Transactions on Signal Processing, 53(11), 4363-4375, 2005.

Sun, L., and Zhao, C.: Optimizing Blind Equalization Intelligent Algorithm for Wireless Communication Systems, 3rd International Conference on Natural Computation, ICNC 2007, 3, 146149, 2007.

Dabeer, O., and Masry, E.: Convergence Analysis of the Constant Modulus Algorithm, IEEE Transactions on Information Theory, 49(6), 1447-1464, 2003

Podilchuk, C.I., and Delp, E.J.: Digital Watermarking: Algorithms and Applications, IEEE Signal Processing Magazine, 18(4), 3346, 2001.

Samee, M. K., and Götze, J.: Increased Robustness and Security of Digital Watermarking Using DS-CDMA, Proceedings of the 7th IEEE International Symposium on Signal Processing and Information Technology, 189-193, 2007.

Samee, M. K., Götze, J., Ruan, S. J., and Pai, Y. T.: Digital Watermarking: Spreading Code versus Channel Coding, Proceedings of the 3rd IEEE International Symposium on Communications, Control and Signal Processing, Malta, 1409-1412, 2008. 